\title{
High Operation Capable Marine Dual Fuel Engine with LNG*
}

\author{
Koichi Watanabe**
}

Niigata Power Systems has been developing the gas engine for about 30 years as one of the best matched products which satisfies the trend of social requirements, such as an environmental preservation and fuel economy, and an electrical demand in industrial field.

Niigata developed new gas engine for marine which is called dual fuel engine used two-type of fuel, oil and gas.

The marine dual fuel engine was designed through diesel design experience and micro pilot technology for diesel mode operation and gas mode operation respectively. Niigata developed the propeller direct drive type dual fuel engine which can meet the desired load operation characteristics coming from the tugboat which works in harbor without generating abnormal combustion such as a knock, with original combustion technology.

Sudden acceleration torque and slowdown torque are required of a tugboat at the time of navigation of a large-sized ship.

Moreover, Niigata Dual fuel engine meets NOx emission level in IMO Tier II and Tier III at diesel operation and gas operation respectively. This performance can be accepted in ECA area.

\section{Introduction}

Niigata Power Systems (NPS) has continuously strived to develop gas engines that meet the needs of the times. In particular, its gas-fueled engines designed for large power generating systems have received favorable reviews not only for high economic efficiency, but also for environmental friendliness and long maintenance period. Among these gas engines is the micro-pilot ignition series developed ten years ago, which achieved a brake mean effective pressure (BMEP) as high as $2.0 \mathrm{MPa}$; systems powered by this series of engines attained electrical efficiency exceeding $43 \%$, the highest in the world among 3 MW class power generation systems at that time. To date, more than 100 of these engines have been delivered (amounting to $226 \mathrm{MW}$ in total output) and are currently operating satisfactorily in Japan. Customers of this engine series have praised its low running cost (requiring maintenance at minimum intervals of 4000 hours), stable operating performance, and high economic efficiency.

NPS recently developed a new gas engine for

\footnotetext{
* Received August 24, 2015

**Niigata Power Systems Co., Ltd.
}

marine that provides even higher economic and environmental performance, and also a marine gas engine featuring superior load-following capability and enhanced environmental design, which is increasingly expected in the marine engine field.

This paper describes this newly developed engine for marine applications.

\section{Gas engine development at NPS}

For the past 30 years, NPS has been fully committed to developing and producing gas engines that meet the demands of the times. Using the diesel engine design with proven reliability as the base, the company succeeded in developing a spark-ignition, lean-burn engine running on $100 \%$ fuel gas and a dual-fuel engine running on both fuel oil and fuel gas (Figure 1).

About 15 years ago, NPS developed and marketed a new gas engine with higher output and performance using a micro-pilot ignition system based on the technologies acquired through the development of the abovementioned engines. The performance of the micro-pilot ignition engine is 
still ranked top in the world. Combined with its excellent economic efficiency, the micro-pilot ignition engine continues to be popular with customers.

In 2009, NPS started working on a new gas engine for marine application aiming at even higher performance, and the project was recently successfully completed.

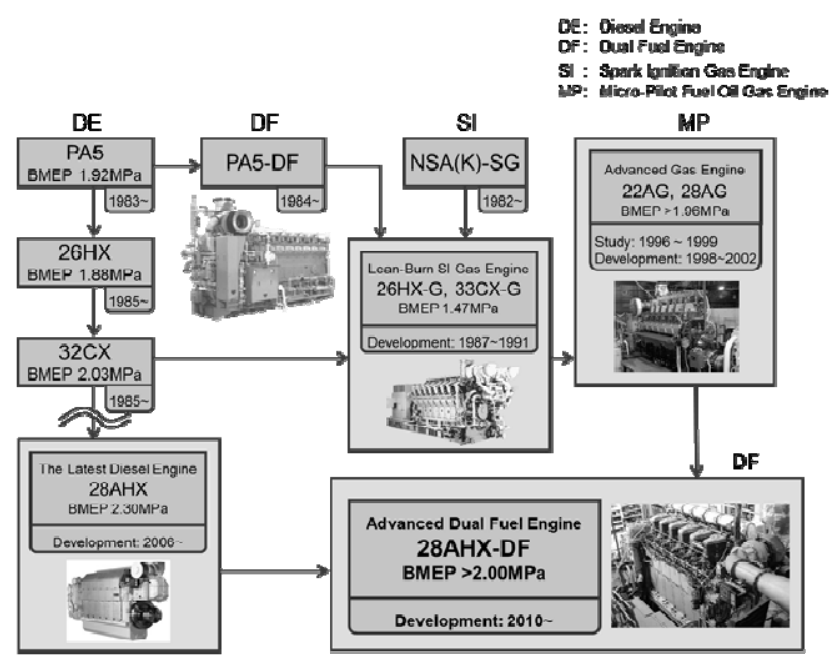

Figure 1 Gas Engine Development at NPS

\section{Trends in gas engine market}

Environmental protection has long been a major concern throughout the world and regulatory organizations are tightening standards. A typical example in the field of marine engines is the Tier III standards of the International Maritime Organization (IMO). However, it is difficult to design a diesel engine that satisfies these standards; additional means are needed to reduce the nitrogen oxide (NOx) emissions, such as an selective catalytic reactor (SCR) device, which is indispensable and presently under study. Additional devices, however, take up space which is limited on a ship.

On the other hand, the fuel burning process in gas engines produces smaller emissions than diesel engines; by nature, exhaust gases from gas engines contain less fuel-derived emissions, as shown in Figure 2. This advantage allows gas engines to satisfy the standards without additional equipment such as an SCR device.
However, the transient characteristics of gas-fueled engines are inferior to than existing marine diesel engine, and so are rarely used for marine applications, even though they have many applications for power generation on land. Therefore, the transient operating characteristics should be improved before gas engines can be used as a power source for propelling ships.

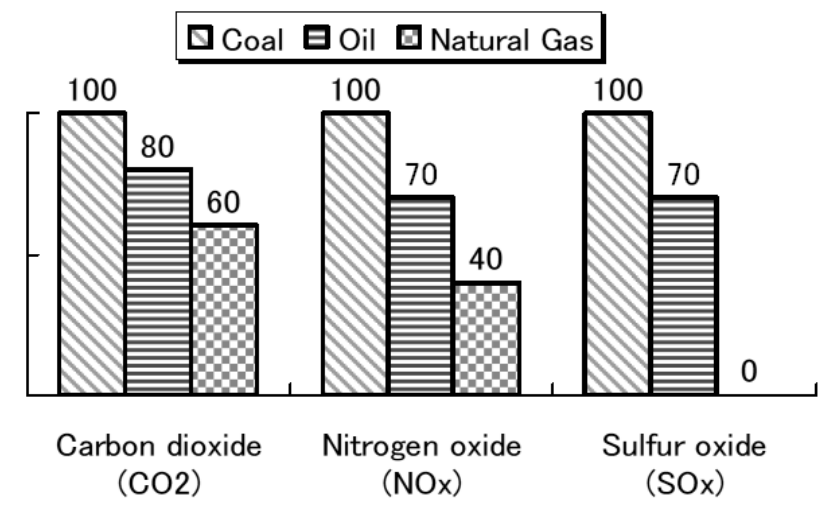

Data : IEA「Natural Gas Prospects to 2010」(1986)

Figure 2 Exhaust Emissions of Different Fuel Types

\section{NPS new marine gas engine (Dual-fuel engine)}

\subsection{Design features and characteristics \\ (related to IMO NOx emission standards)}

NPS continues to develop gas-fueled engines for marine applications based on the dual-fuel engine, which can operate as an ordinary diesel engine and also as a gas engine. For example, even if one of the gas combustor components malfunctions while operating in the gas mode, the ship can continue running by switching the engine to the diesel mode. This capability helps reduce the risk of drifting due to loss of control in the event of a problem. In other words, the dual-fuel engine provides redundancy for the ship's propulsion system, which is one of the most important features for safety operation. Table 1 shows the specifications of the NPS dual-fuel engine and Figure 3 shows a general view of its prototype. 
Table 1 Specifications of NPS Marine Gas Engine

\begin{tabular}{|l|l|}
\hline Item & Specification \\
\hline Engine & 28AHX-DF \\
\hline $\begin{array}{l}\text { Combustion } \\
\text { system }\end{array}$ & $\begin{array}{l}\text { Micro-pilot ignition \& lean-burn } \\
\text { system }\end{array}$ \\
\hline B.M.E.P & $2.0 \mathrm{MPa}$ \\
\hline Fuel gas & Vaperized LNG \\
\hline Ignition fuel oil & Marine diesel oil (MDO) \\
\hline
\end{tabular}

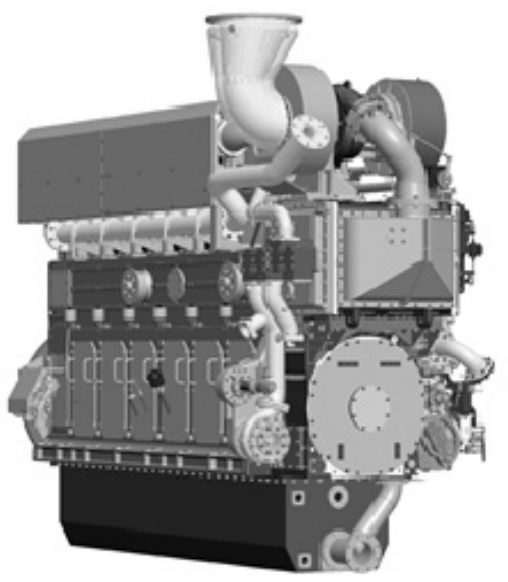

Figure 3 General View of Dual-fuel Engine Prototype

The design concept of this marine gas engine is the two engine operation modes. The diesel mode is used for starting, an emergency and stopping, while the gas mode is used for all other operating phases, typically cruising, as schematically shown in Figure 4. By switching the operation mode according to this scheme, it is possible to maximize the operating time in the gas mode and thus satisfy the design concept of clean emission ship. Figure 5 shows a time-series typical operational cycle of the engine, with time on the horizontal axis. Figure 6 shows comparison of emissions between gas and diesel operation.

Potential emergencies include abnormal combustion inherent to the gas engine (such as knocking), trouble with the electronic fuel control system and fuel shortage.

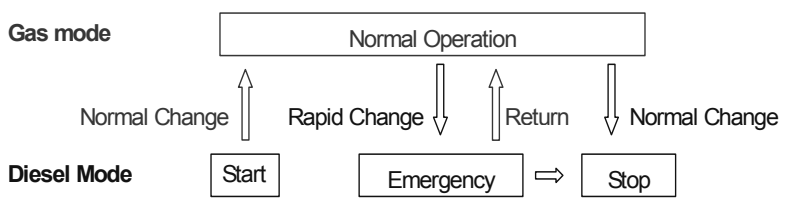

Figure 4 Shifts in Operation Mode of Marine Gas Engine (Schematic)

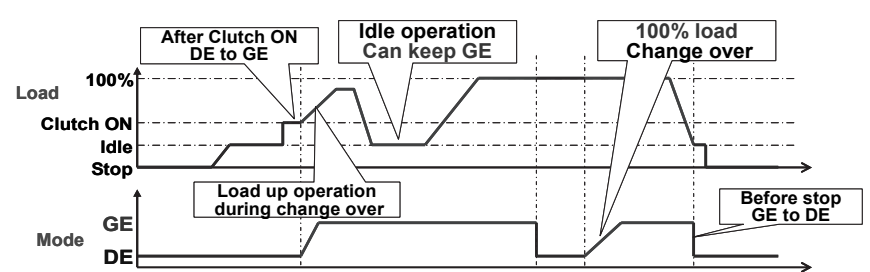

Figure 5 Shifts in Operation Mode of Marine Gas Engine (Chart)

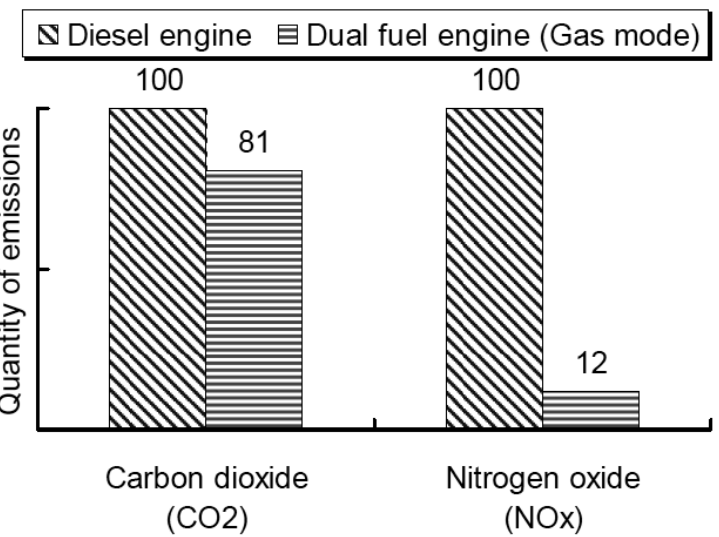

Figure 6 Comparison of Emissions with Operation Mode

The target set for $\mathrm{NO}_{\mathrm{x}}$ emissions for this engine is to meet the IMO Tier III standard for the gas mode operation and the IMO Tier II standard for the diesel mode operation. Table 2 shows the required values of these standards and the measured values actually achieved by the engine.

Table $2 \quad \mathrm{NO}_{\mathbf{x}}$ Emission Measurements in Diesel and Gas Engine Operation Modes

\begin{tabular}{|l|l|l|}
\hline Operation Mode & Diesel Mode & Gas Mode \\
\hline IMO NOx & IMO Tier II & IMO Tier III \\
\hline Regulation value & $\leqq 9.6 \mathrm{~g} / \mathrm{kWh}$ & $\leqq 2.4 \mathrm{~g} / \mathrm{kWh}$ \\
\hline Measured value & $9.6 \mathrm{~g} / \mathrm{kWh}$ & $0.9 \mathrm{~g} / \mathrm{kWh}$ \\
\hline
\end{tabular}

\subsection{Quick load change operation}

As mentioned earlier, there is a problem that must be solved before gas engines can accommodate rapid changes in load. When the engine on a ship directly drives the propeller, its speed is changed according to the change in load, as shown in Figure 7. 
During lower loads, the engine speed is slow, and a small amount of low-temperature exhaust gases is released. This leads to sluggish operation of the turbocharger since it is driven by the energy in the flow of exhaust gases. Under these operating conditions, the turbocharger cannot supply a sufficient amount of air to keep the air-fuel ratio in the appropriate zone, and as a result, the engine may fail to continue running due to knocking or other abnormal combustion state.

On the other hand, when the engine is operated with the load rapidly changing, the turbocharger's speed may fail to rise following an increase in load, creating some load zones where adequate amounts of air are not supplied to the engine and eventually for causing the engine to knock. A typical case of this condition is depicted in Figure 8.

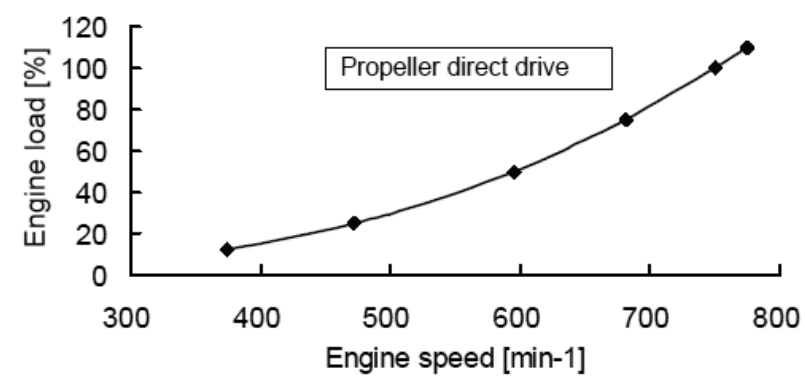

Figure 7 Engine Speed vs. Load (Case of Propeller Directly Driven by Engine)

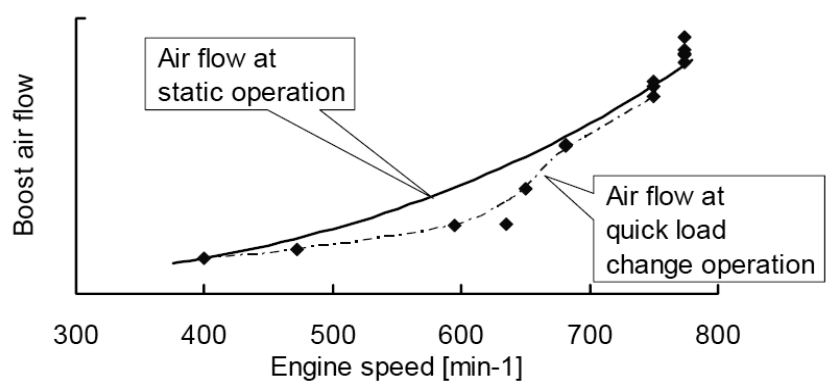

Figure 8 Engine Operation with Insufficient Air Supply

To overcome these shortcomings, the newly developed gas engine employs the common rail, which enables variable control according to changing load conditions.

To control these mechanisms, NPS developed a high-performance control system and succeeded in achieving stable engine operation under low loads and proper load-following capability under rapid load changes by incorporating this controller in the engine. Figure 9 presents an example of the results, showing that a rise in load factor from $15 \%$ to $100 \%$ can be completed in approximately 15 seconds.

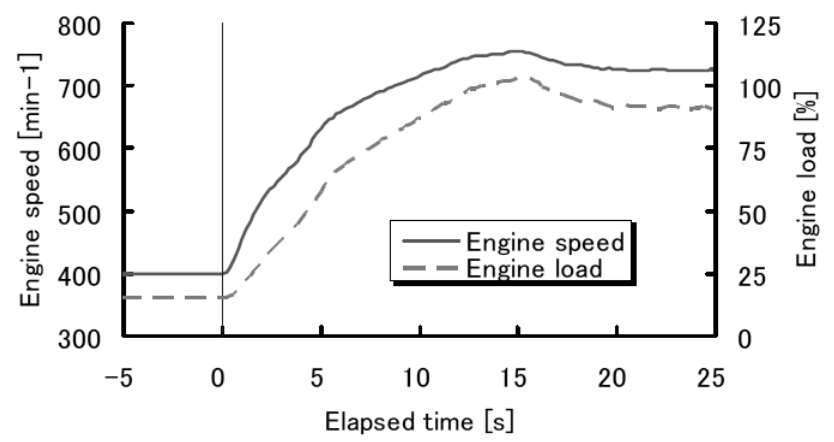

Figure 9 Improved Characteristics at Rapid Load Change

In addition, the control system is optimized for instant switching of engine operation from gas mode to diesel mode and vice versa to be able to deal with emergency situations. An example of switching the engine operation mode is shown in Figure 10 and the load factor ranges where mode switching is possible. The control system is adjusted so that switching from diesel to gas mode is possible all operation load and the operation load of gas mode is between $0 \%$ and $100 \%$ load.

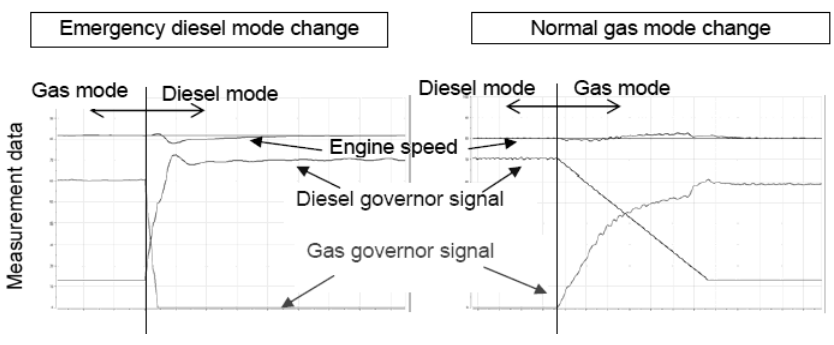

Figure 10 Switching of Engine Operation Mode

\section{Application to tugboats}

The IMO Tier III standards will come into force in 2016 in Emission Control Areas (ECAs). This has led many tugboat operators to consider introducing gas engines that can satisfy the environmental standard requirements without using additional equipment. 
Applying gas engines to tugboats has so far been considered impracticable because, as already seen, it is difficult for gas engines to follow frequent and rapid load changes, which are unavoidable in tugboat operation.

However, NPS has solved this problem by taking the technical approaches discussed earlier and succeeded in developing gas engines appropriate for propelling tugboats.

NPS has been producing as its main product the medium-speed diesel engines combined with the Z-propeller propulsion system for tugboat applications and has delivered many of these units.

The company intends to meet customers' demands by supplying a gas-engine-powered version of the unit and is developing a gas-fueled engine optimized for tugboat applications.

Figure 11 shows the gas-fueled engines installed on a tugboat.

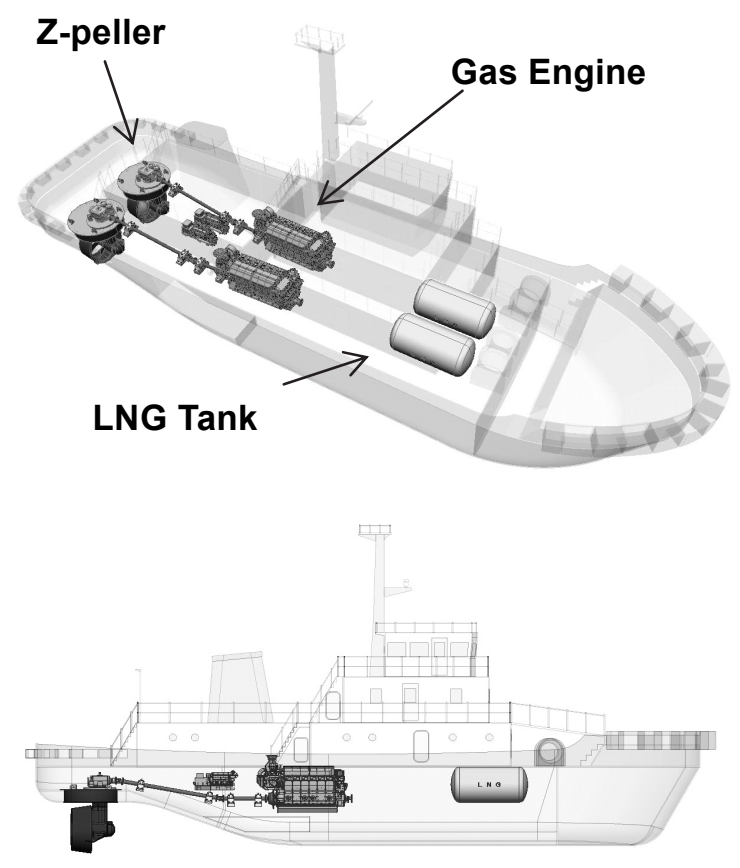

Figure 11 Gas-Fueled Engines on Tugboat

Figure 12 shows a typical operation pattern of an ordinary diesel-engine-powered tugboat.

It indicates frequent changes in speed (load) including a rapid change from the idling speed (400 $\left.\min ^{-1}\right)$ to the rated speed $\left(750 \mathrm{~min}^{-1}\right)$ in 15 seconds.
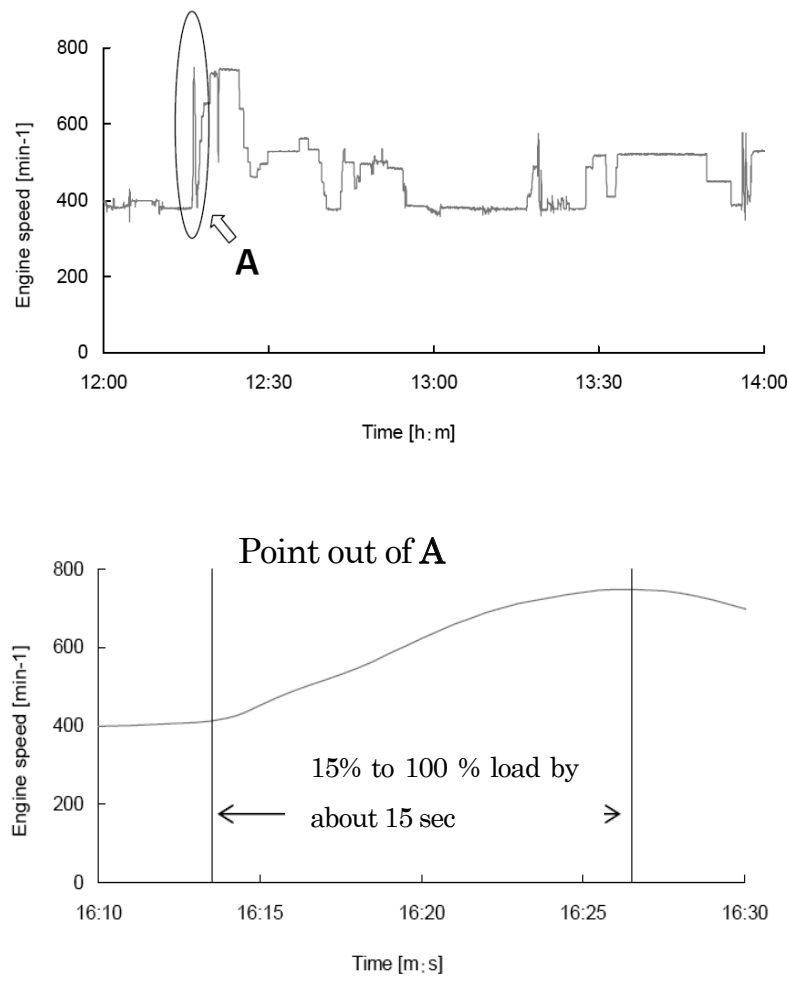

Figure 12 Typical Operation Pattern of Tugboat

To ensure that the gas engine properly follows the rapid load changes experienced in tugboats, NPS employed different types of variable control systems and optimized them to meet the objective.

Figure 13 shows the outcome of the development; the engine is well adapted to rapid load changes as seen in the operation pattern of ordinary tugboats.

Thus, the gas-fueled marine engine developed by NPS is suitable for tugboat applications.

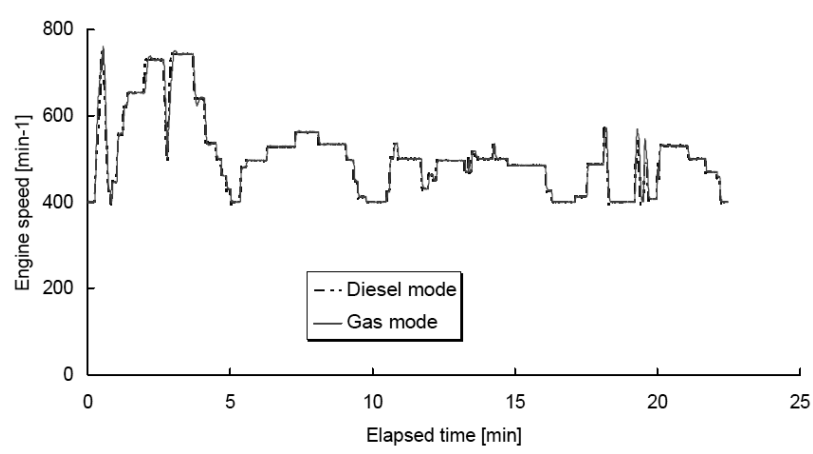

Figure 13 Outcome of development 


\section{Conclusion}

NPS has been developing and supplying gas engines that meet the demands of the times. It recently completed the development of the 28AHX-DF, a dual-fuel gas engine with a high level of economic efficiency, environmental friendliness and safety.

For marine gas engines which must follow frequent changes in load, NPS has developed a new control system consisting of a multiple number of variable control mechanisms and a high-performance controller for controlling these mechanisms.

The system ensures that the engine operates stably under low loads and follows rapid load changes.

Through tests conducted by simulating the operation pattern of a diesel engine powered tugboat, the new marine gas engine adopting this control system has demonstrated improved operating characteristics comparable to those of the diesel engine.

NPS will continue to offer engines with the most suitable combustion systems for individual marine applications in response to each customer's requirements while seeking higher performance of the reciprocating engine.

\section{Acknowledgements}

The Dual Fuel marine propulsion engine 6L28AHX-DF introduced today uses part of technology from the research development which was selected as a supported project of "Research project of $\mathrm{CO} 2$ reduction from marine vessels" by Ministry of Land, Infrastructure, Transport and Tourism, selected as a supported project by Nippon Kaiji Kyoukai(Class NK), selected as a joint research with Japan Ship Technology research association and financially supported by the NIPPON Foundation.

NIIGATA expresses sincere appreciation to these associations and foundation.

\section{References}

[1] Goto, S., et al., "Development of Advanced Gas Engine 22AG with High BMEP, High Efficiency, Ignited by Micro-pilot MDO"; 23rd CIMAC Paper pp941-956, (2001)

[2] Takahashi, S., et al., "Result in Service Operation of 1.3 MW Micro-pilot Gas Engine, and its further Development"; 24th CIMAC, Paper No. 146, (2004)

[3] Goto, S., et al., "Study of higher adaptability in 1 to 3 MW class micro-pilot gas engine for co-generation field through its development work and field experience" ; 25th CIMAC, Paper No. 111, (2007)

[4] Nakayama, S., et al., "Experiences on 1 to $6 \mathrm{MW}$ class higher adaptable micro-pilot gas engines in one hundred fields and over fifty thousand running hours"; 26th CIMAC, Paper No. 125, (2010)

[5] Watanabe, K., et al., "Advanced development of medium speed gas engine targeting to marine and land"; 27th CIMAC, Paper No. 99, (2013) 